\section{Automatic recognition of sleep spindles using short-term spectral analysis*}

\author{
B. VO-NGOC, D. POUSSART, and J. M. LANGLOIS \\ Département de génie électrique et Centre de Recherche en Bionique \\ Université Laval, Quebec, Canada
}

An approach to recognize automatically sleep spindles by using short-term spectral analysis is described. The EEG signal is sampled and stored on a magnetic tape in digital form. The data is analyzed on a PDP-8 computer, and the result can be either displayed on an oscilloscope or stored on another magnetic tape for further analysis. The program is written in conversational form and provides the operator with the ability to change any parameter in the program quickly by a simple command on the Teletype keyboard. of the spectra are uncalibrated). It can be noted that when spindle activity is absent, the spectrum shows only very low frequency components $(0$ to about $5 \mathrm{~Hz}$ ), as illustrated, for example, in Spectra Nos. 1 and 12. However, whenever spindle activity is present, an increased power around $10 \mathrm{~Hz}$ is readily visible as a well-defined peak on the corresponding spectrum (for example, in Spectrum No. 8). A small increase around $20 \mathrm{~Hz}$ can be noted also, as well as (more occasionally) a third peak, between 25 and $30 \mathrm{~Hz}$. These "harmonjcs" of the fundamental frequency presumably reflect the
It is well known that sleep spindles, consisting of bursts of large rhythmic oscillations (rate of about 10-12/sec) of waxing and waning amplitude, appear during drowsy state or light sleep. However, the precise origin of this type of activity is still uncertain.

The source of cortical spindle activity was first attributed to the midline nuclei of the thalamus by Dempsey and Morison (1942). Hess, Koella, and Akert (1953) have, rather, suggested that spindles arise from varying sites in the cerebral cortex. The recent work of Anderson and collaborators $(1967,1968)$, however, has revived the notion of thalamic origin. Although the midline nuclei do not appear to be necessarily involved, since their removal has no significant effect on the appearance of spindles, these workers strongly believe that spindle activity is driven from multiple independent pacemaker areas in the thalamus.

Most of the work reported so far has involved only visual examination and one-to-one comparison of spindles recorded in different cortical and subcortical locations. The scheme of automatic spindle detection, described below, was designed to facilitate the measurement of the stochastic characteristics of spindles, such as duration, relative time of occurrence in different regions, and correlation.

\section{METHOD}

The relatively high amplitude and particular frequencies of oscillations of sleep spindles suggest that a method of detection based on spectral analysis might yield useful results.

Part a of Fig. 1 shows a segment of EEG data, recorded from the frontal region of the cortex of cat, which includes a typical spindle. Corresponding short-term power density spectra are shown in Part c of the figure (note that the vertical scales

*Aspects of this work were presented at the 1970 Spring DECUS Symposium, Atlantic City.
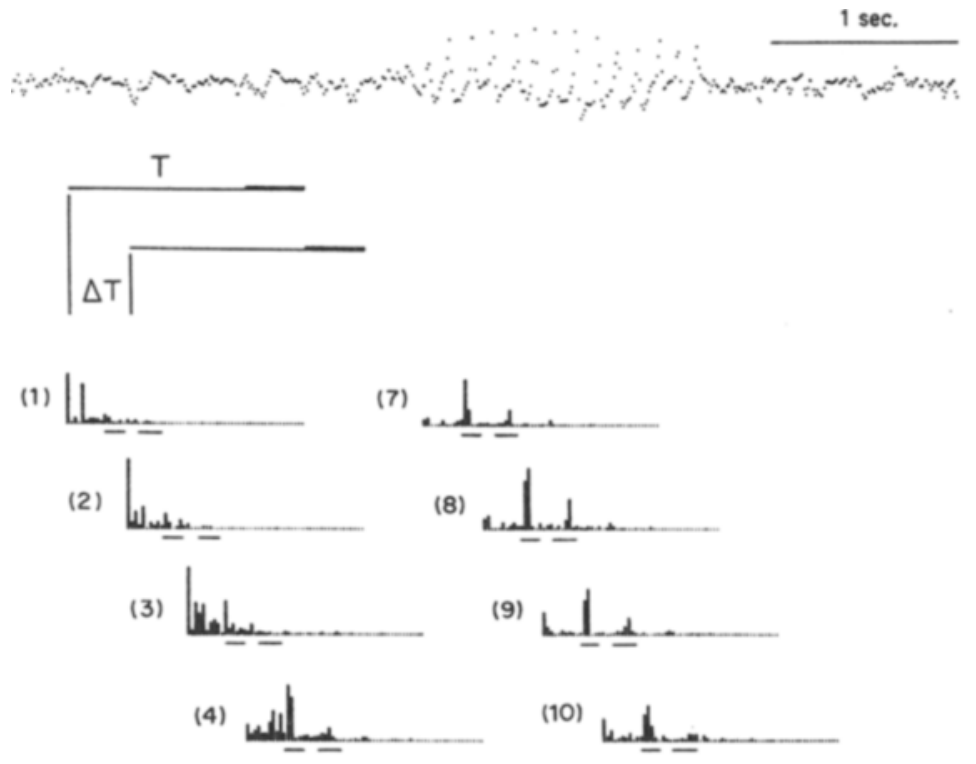

(5)

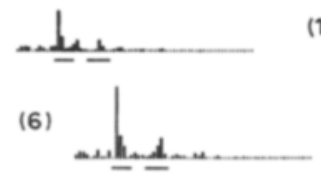

(11)
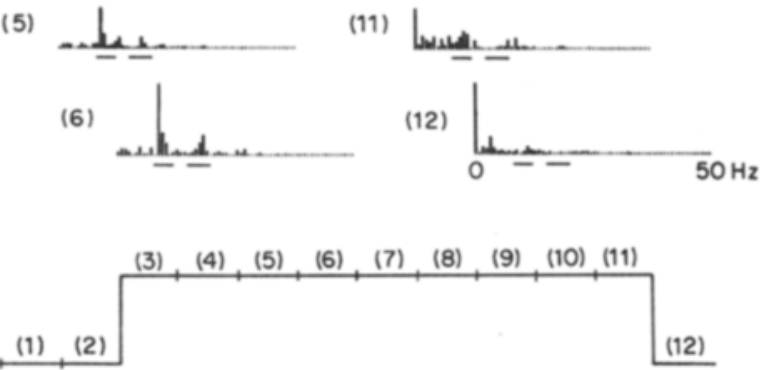

(d)

Fig. 1. (a) A segment of EEG data recorded from the frontal region of the cerebral cortex of cat during a barbiturate sleep (Pentothal, dose $10 \mathrm{mg} / \mathrm{kg}$ ) which shows spindle activity. (b) The overlapping segments illustrate how the data are taken for analysis vs real time. The decision obtained on each segment of data of length $T$ is assigned to the end portion of length $\Delta T$ of that segment, as shown by the heavy bars. (c) Power spectra computed for overlapping segments of the above EEG data. Each spectrum corresponds to the segment of data directly above. Here $\mathrm{T}=1.28 \mathrm{sec}, \Delta \mathrm{T}=320 \mathrm{msec}$, and the frequency ranges linearly from 0 to $50 \mathrm{~Hz}$. The vertical scales are linear and uncalibrated. The frequency band limits used for deciding on the presence of spindles are shown by the bars underlining the spectra and extend from 8 to 12 and from 15 to $20 \mathrm{~Hz}$. One can note that as the spindle develops, the power in these bands becomes appreciable. (d) The output of the program for spindle detection. The plateau corresponds to the presence of a spindle. Each decision has a number which corresponds to the spectrum with the same number in Part $c$. The detection threshold is set at $1 / 3$. 


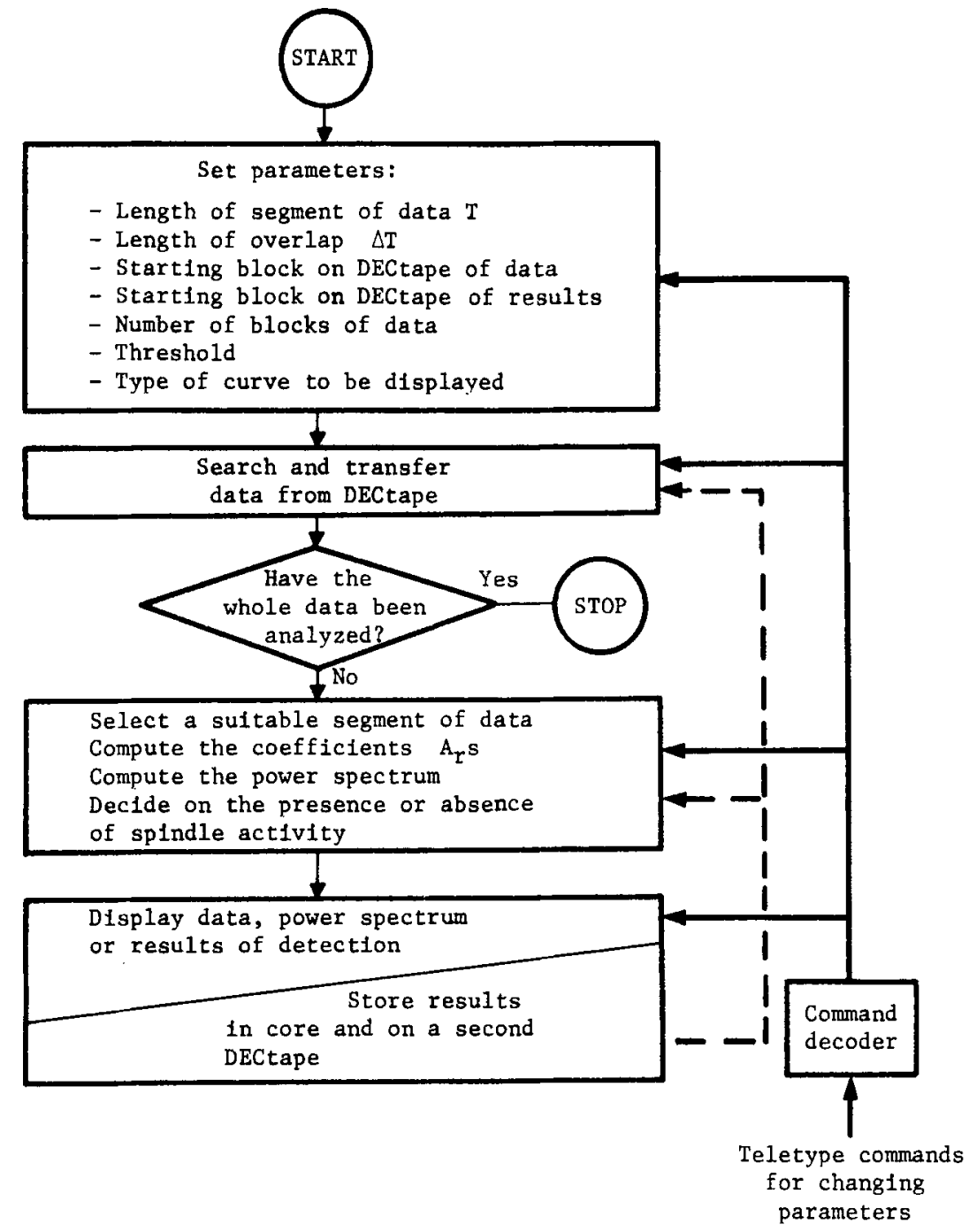

Fig. 2. Schematic flow diagram of the program for the automatic detection of sleep spindles. In the interactive mode (full lines), the parameters of analysis are entered via the Teletype. Once satisfactory values of these parameters have been determined, a suitable command will switch the program to the automatic mode (dashed lines), and the program will analyze the whole data.

"spiky" shape of the spindle oscillations.

The qualitative difference between the spectra measured in the absence or presence of spindle activity has suggested the following detection criterion: if the ratio of the power contained in two frequency bands centered around 10 and $20 \mathrm{~Hz}$ over total power exceeds a certain threshold value, spindle activity is declared present and, otherwise, absent in that segment of data. The threshold value is to be determined by trial and error on a learning set, so that an acceptable compromise between false and missed detections can be achieved.

We wish to determine automatically not only the presence and duration of spindles, but also their time of onset, in order to look for systematic differences of latency between by its samples taken at consecutive time intervals, $\Delta t \leqslant 1 / 2 f_{\max }$ sec. In the present investigation, the frequencies of import extend from 0 to $25 \mathrm{~Hz}$ and we have accordingly chosen a sampling rate of $100 / \mathrm{sec}$, i.e., $\Delta t=10 \mathrm{msec}$.

In view of the variation of a few hertz, which appears to normally occur in the basic frequency of spindles, one might expect a frequency resolution $\Delta f$ of $1 \mathrm{~Hz}$ to be adequate. This value of $\Delta \dot{f}$ requires a length of data $T$ of at least 1 sec. This duration, however, is comparable to that of a spindle, and a detection method using spectra computed for consecutive disjoint lengths of data would probably prove inadequate for the present purposes.

The temporal resolution in the detection of the onset of a spindle can presumably be improved by evaluating the spectra over segments of data which overlap by a length $\Delta T$, as illustrated in Part b of Fig. 1. Each decision, computed on a segment of data of length $T$, is then assigned to the end portion of length $\Delta T$ of that segment. Qualitatively, this operation is the digital counterpart of the analog processing which would be achieved by a bank of bandpass filters whose squared outputs would be applied to "amnesic" integrators with time constant $\mathrm{T}$, sampled at time intervals $\Delta \mathrm{T}$, and compared according to a set threshold. Although the onset of a spindle can then be detected within a time resolution $\Delta T$, at least in principle, the somewhat random character of spindle activity suggests that there is but little to gain by making $\Delta \mathrm{T}$ very short (with the cost of unnecessary computation). Values of $\Delta \mathrm{T}$ in the range of $0.2 \mathrm{sec}$ are presently used as a practical compromise.

This approach requires a great deal of power spectrum computation and is only practical because of the efficiency of the fast Fourier transform algorithm [see Bergland (1969) for a general introduction to FFT and Dumermuth \& Fluhler (1967) for a typical example of previous application to EEG analysis ]. The FFT algorithm is an efficient procedure for computing the coefficients $A_{r} s$ of the discrete Fourier transform:

$$
\Delta \mathrm{f} \approx \frac{1}{\mathrm{~T}}=\frac{1}{\mathrm{~N} \Delta \mathrm{t}}
$$

where $\mathrm{N}$ is the number of samples and $\Delta t$ is the sampling time interval used when the original data is digitized.

On the other hand, the interval $\Delta t$ must satisfy the sampling theorem (for example, see Tou, 1959), which states that if a signal $f(t)$ has a frequency spectrum extending from zero to $f_{\max } \mathrm{Hz}$, it is completely determined

$$
\begin{gathered}
A_{r}={ }_{k=0}^{N-1} X_{k} \exp (-2 \pi j r k / N) \\
r=0,1, \cdots, N-1 .
\end{gathered}
$$

where $X_{k}$ is the $k^{\text {th }}$ sample of a time series of $N$ samples and $j=\sqrt{-1}$. In this case, the $X_{k} s$ are real numbers since they represent data samples and the $A_{r} s$ are complex. The discrete power density spectrum is then simply 
evaluated from the expression:

$$
A_{r}{ }^{2}=\left(\operatorname{Re} A_{r}\right)^{2}+\left(\operatorname{Im} A_{r}\right)^{2}
$$

where Re and Im denote the real and imaginary parts, respectively.

\section{IMPLEMENTATION AND RESULTS}

The main hardware used in the present study consists of a PDP-8 computer (Digital Equipment Corporation), with $8 \mathrm{~K}$ memory, extended arithmetic element, DECtapes, analog-digital converter, programmable clock, CRT display, point plotter, and a Honeywell 7600 FM magnetic tape recorder.

The EEG analog signal originally recorded on FM magnetic tape is first digitized (10-bit resolution and rate of 100 samples/sec) and then transferred onto DECtape. Storing the EEG signal on digital magnetic tape is desirable since the same precise data can be repeatedly retrieved while parameters are changed during the subsequent analysis.

Spindle detection is performed by means of a program which includes a FFT subroutine (Rothman, 1968) for computing the coefficients $A_{r}$ s.

In order to facilitate the evaluation of the method, the initial portion of the program (see Fig. 2) is interactive. The main parameters of analysis, the address of the data, the number of samples $N$ corresponding to the desired $\mathrm{T}$, the overlap $\Delta \mathrm{T}$, the value of the detection threshold, and the upper and lower limits of the two frequency bands of interest, can be changed simply from the Teletype keyboard. The program then inputs the requested data, computes the power spectrum, determines whether spindle activity is present or absent, and waits for a suitable command from the Teletype to proceed to the next segment of EEG. The EEG data, its spectrum (with chosen frequency limits), and the result of the detection are continuously displayed on the CRT.

Once satisfactory values of the parameters have been determined on a training set, the program is set to an automatic mode. It examines the entire data and stores the results on a second DECtape for further analysis. In essence, then, the program converts raw EEG data into a rectangular wave whose plateaus presumably correspond to the presence of spindles.

The illustrative results presented in Fig. 1 are obtained from the EEG recorded from a cat during barbiturate sleep (Pentothal, dose $10 \mathrm{mg} / \mathrm{kg}$ ). In this case, $\mathrm{T}$ is $1.28 \mathrm{sec}(\mathrm{N}=128)$ and $\Delta \mathrm{T}$ is $320 \mathrm{msec}$. The frequency limits, as shown by the bars underlining the spectra, extend from 8 to 12 and from
15 to $20 \mathrm{~Hz}$, and the detection threshold is set at $1 / 3$. From each of these spectra, the program decides upon the presence or absence of spindle activity according to the chosen criterion, and Part $d$ of the figure shows the final result. Clearly, the program correctly detects the onset of spindle activity. However, since the decision is assigned to the end portion $\Delta \mathrm{T}$ of the segment $\mathrm{T}$, as described previously, a positive indication for the presence of spindle persists after spindle activity has ceased. Thus, the estimated duration of a spindle is consistently too long by a time interval of the order of $\mathrm{T}-\Delta \mathrm{T}$. This systematic bias can be taken into account in subsequent analysis.

The speed of the program depends mainly on the number of samples $\mathrm{N}$ used for computing each spectrum and the number of required spectra, i.e., the time overlap $\triangle T$. For $N=128$ samples and a shift of 16 samples, i.e., $\Delta \mathrm{T}=0.16 \mathrm{sec}$, the program performs the analysis almost in real time.

This program occupies practically all of the lower $4 \mathrm{~K}$ of memory. This includes the spaces for the various input-output subroutines and temporary storage.

\section{DISCUSSION}

Because of the somewhat operational definition of a spindle, it has not been possible to assess the performance of the present program in quantitative terms. False or missed detections do occur occasionally. However, in most instances, the results given by the program have coincided closely with the subjective judgment of the $E$, even in the case of relatively high background activity.

It seems that missed detections are often caused by the simultaneous presence of very low-frequency, high-amplitude components, which momentarily increase the total energy in the signal. It has been found that this problem could be avoided by completely disregarding low-frequency components (e.g., below $2 \mathrm{~Hz}$ ) in the computation of the detection criterion described above.

One might note that the high amplitude of the EEG during spindle activity, when compared to that of the background, is not fully exploited. The detection criterion was purposely chosen to be based on relative rather than absolute power in order to avoid tricky calibration problems (especially during the simultaneous analysis of several EEG recordings).

Furthermore, since one can assign constraints on the accepted duration of a spindle, such as, say, 0.3 to $4 \mathrm{sec}$, the overall performance can be improved by rejecting detections exceeding these limits. This can be very easily implemented during further analysis.

Programs which compute various statistics on the random square wave that is produced by the present method have been developed and are being used to study spindle activities. In particular, the distribution of the duration of spindles and of interspindle intervals during various stages or types of sleep is being investigated. A version of the present program which simultaneously analyzes two EEG channels has also been developed. It is being used at present in a study of the correlation between spindle activities recorded from different sites of the cortex and subcortical structures.

Obviously, this detection could also be performed simply by analog means, using bandpass filters and comparators, as we tried initially with circuits such as proposed by Lowenberg and McCullough (1963). However, with all the advantages that a digital computer presents over the analog method, such as great control flexibility, memory, precision, and capability for further computation of various stochastic parameters, the digital method is undoubtedly preferable when a small dedicated digital computer is available in the laboratory.

\section{REFERENCES}

ANDERSEN, P., ANDERSSON, S. A.. \& LOMO. T. Some factors involved in the thalamic control of spontaneous barbiturate spindles. Journal of Physiology, 1967, 192, 257-281.

ANDERSEN, P., ANDERSSON, S. A Physiological mechanisms of the alpha waves. In P. Kellaway and I. Petersen (Eds.), Clinical electroencephalography of children. New York: Grune, 1968. Pp. 31-48.

BERGLAND, G, D, A guided tour of the fast Fourier transform. IEEE Spectrum. July $1969,41-52$

DEMPSEY, E. W., \& MORISON, R. S. The interaction of certain spontaneous and induced cortical potentials. American Journal of Physiology, 1942, 135 , 301-308.

DUMERMUTH, G., \& FLUHLER, H. Some modern aspects in numerical spectrum analys of multichannel electroencephalographic data. Medical \& Biological Engineering, 1967, 5, 319-331.

HESS, R JR KOELLA, W. P \& AKERT, $K$. Cortical and subcortical recordings in natural and artificially induced sleep in cats. Electroencephalography \& Clinical Neurophysiology, 1953, 5, 75.90.

LOWENBERG, E, C., \& McCULLOUGH. C. E. An improved high-Q bandpass filter for e $l$ e c t roe $n$ c e p a lo graph y Electroencephalography \& Clinical Neurophysiology, 1963, 15, 706-708.

ROTHMAN, J. E. FFTS-R. A fast Fourier transform subroutine for real valued functions. Digital Equipment Computer Users' Society, 1968, DECUS No. B-143. TOU, J. T. Digital and sampled data contro systems. New York: McGraw-Hill. 1959. 